\title{
vegIMPACT
}

\section{Handbook of vegetable production and economics in Indonesia}

Marcel van der Voort, Herman de Putter, Huib Hengsdijk, Witono Adiyoga

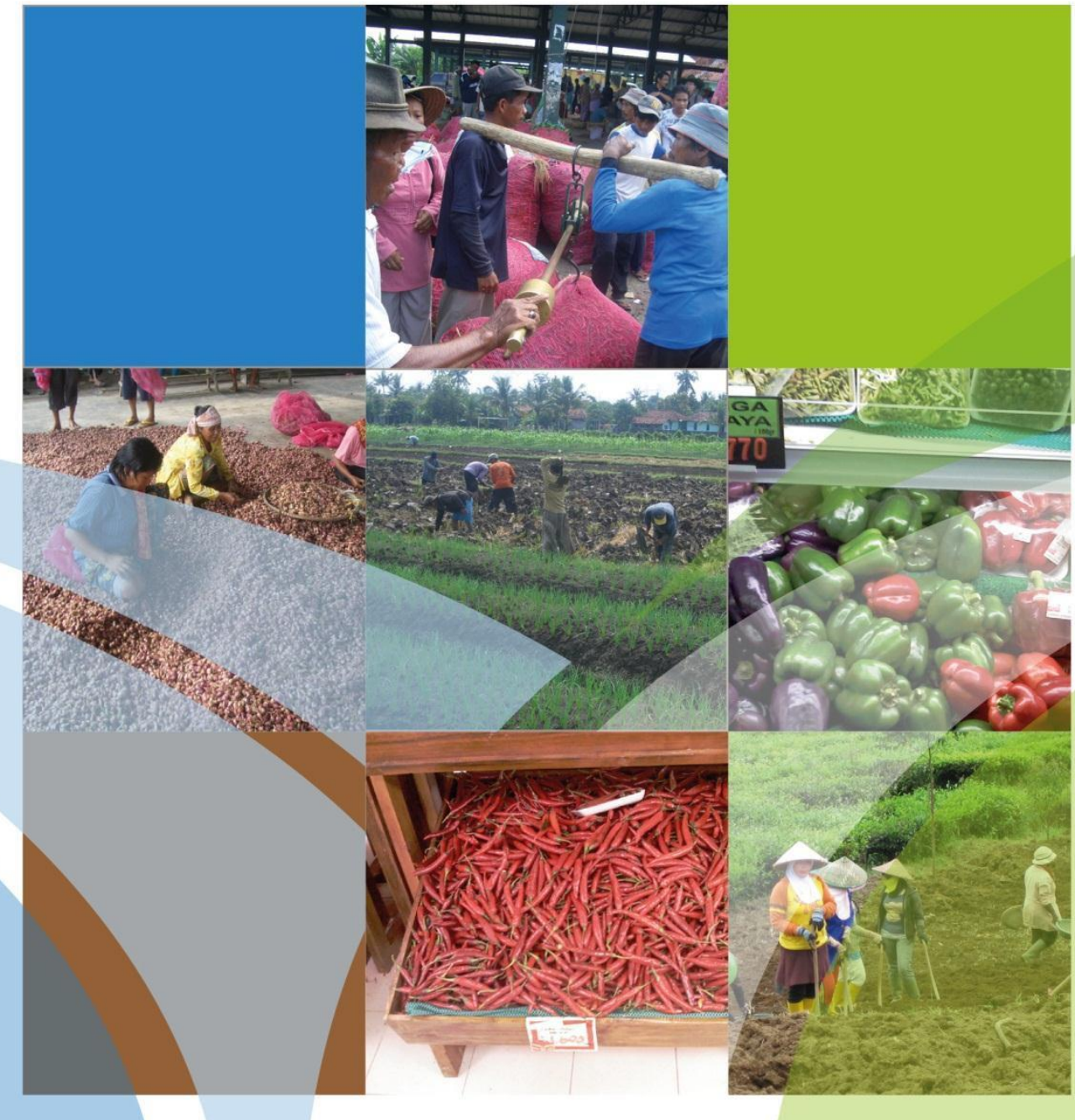

\section{vegIMPACT}

Improved Vegetable Production and Marketing for small farmers to Increase the Food Security status and to promote Private Sector Development in Indonesia 


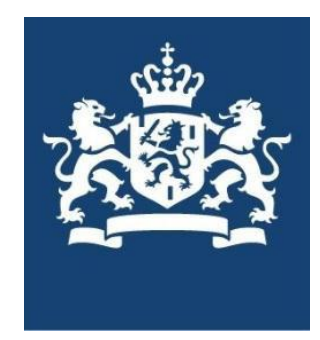

vegIMPACT is a program financed by The Netherlands' Government promoting improved vegetable production and marketing for small farmers in Indonesia, contributing to the food security status and private sector development in Indonesia. The program builds on the results of previous joint IndonesianDutch horticultural development cooperation projects and aligns with recent developments in the horticultural private sector and retail in Indonesia. The program activities $(2012-2016)$ include the Development of Product Market Combinations, Strengthening the Potato Sector, Development of permanent Vegetable Production Systems, Knowledge Transfer and Occupational Health.

Wageningen University \& Research, The Netherlands:

- Wageningen Plant Research, Lelystad

- $\quad$ Wageningen Centre for Development Innovation (CDI), Wageningen

- Wageningen Plant Research, Wageningen

- $\quad$ Wageningen Economic Research, Den Haag

Wageningen University \& Research

Contact person: Huib Hengsdijk, huib.hengsdijk@wur.nl

Indonesian Vegetable Research Institute (IVEGRI, Indonesia)

Contact person: Witono Adiyoga, balitsa@balitsa.org

Fresh Dynamics (Indonesia)

Contact person: Marcel Stallen, info@freshdynamics.biz

Website: www.vegIMPACT.com

https://doi.org/10.18174/472935

\footnotetext{
(C) 2018 Wageningen University \& Research, Wageningen Plant Research, P.O. Box 16, 6700 AA Wageningen, The Netherlands; T +31 (0)317 4807 00; www.wur.nl/plant-research.

Stichting Wageningen Research. All rights reserved. No part of this publication may be reproduced, stored in an automated database, or transmitted, in any form or by any means, whether electronically, mechanically, through photocopying, recording or otherwise, without the prior written consent of Stichting Wageningen Research.
}

DLO is not liable for any adverse consequences resulting from the use of data from this publication. 


\title{
Handbook of vegetable production and economics in Indonesia
}

\author{
Marcel van der Voort, Herman de Putter, \\ Huib Hengsdijk, Witono Adiyoga
}


Table of Contents

Preface

Preface

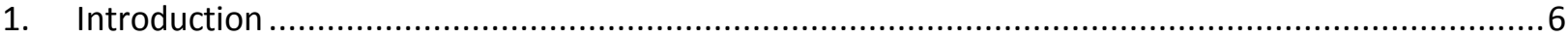

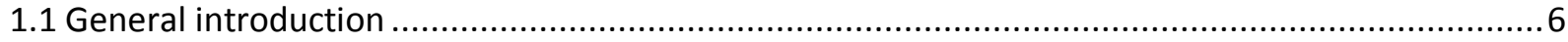

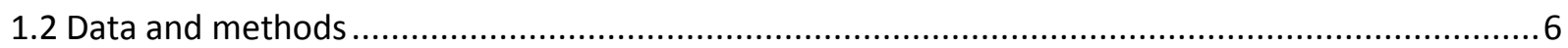

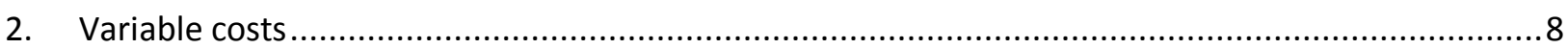

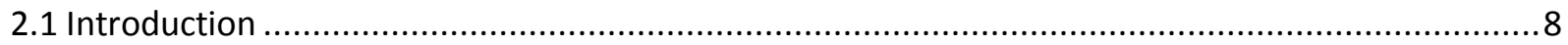

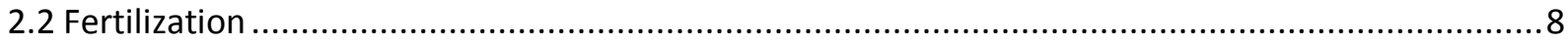

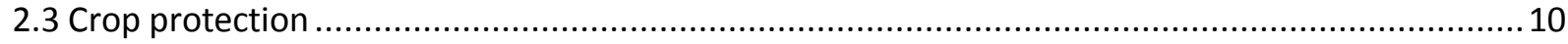

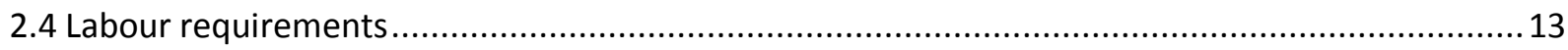

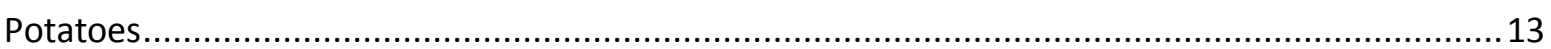

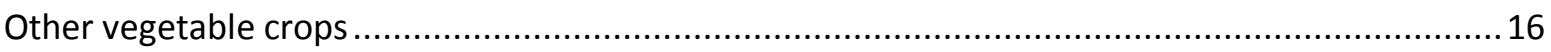

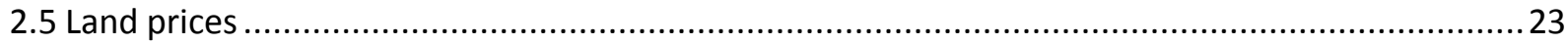

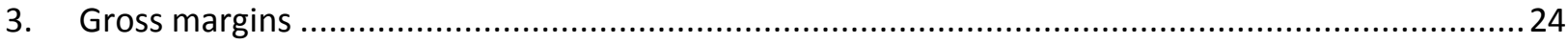

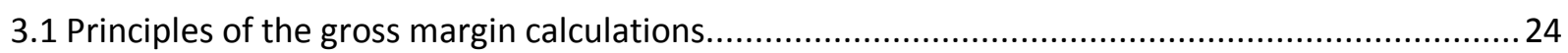

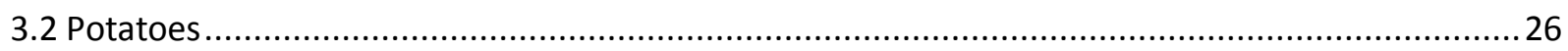

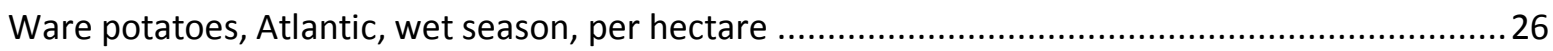

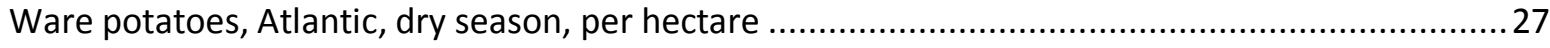

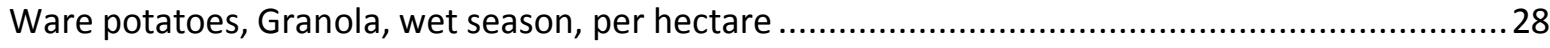

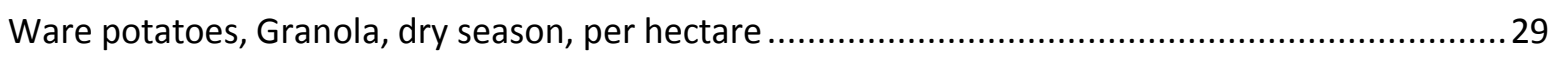

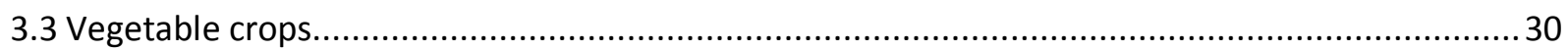

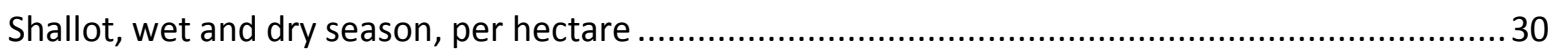

Sweet corn, wet and dry season, average per hectare …............................................................. 31

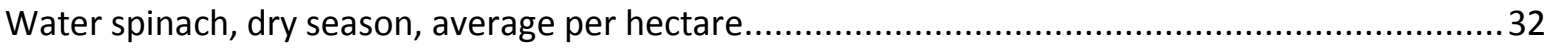

Yard Long Bean, wet and dry season, average per hectare .......................................................... 33

Hot pepper, wet season, average per hectare ....................................................................... 34

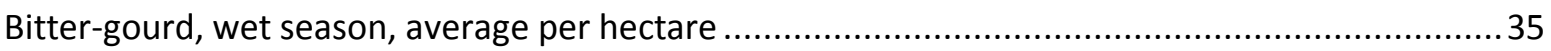

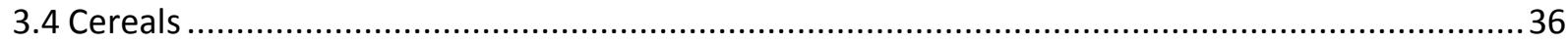

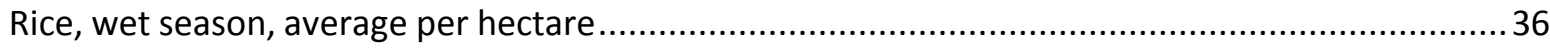

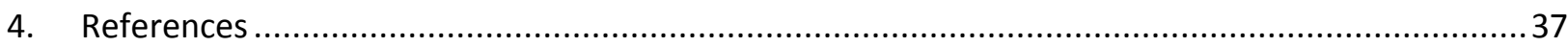




\section{Preface}

The vegIMPACT program, short for 'vegetable production and marketing with impact', aims to improve vegetable production and marketing of small farmers in Indonesia. VegIMPACT contributes to increased food security and private sector development in Indonesia. The program is financed by the Dutch government and is carried out by Wageningen University and Research Centre (WUR) together with local partners and national and international companies in vegetable production and marketing. VegIMPACT carries out a range of intervention strategies, many of which focus on practical training and capacity building of farmers and other stakeholders in vegetable chains.

During program implementation we learned about the high demand in Indonesia for standard and credible horticultural information. For example, information on crop diseases and pesticides as compiled in vegIMPACT's external report no. 10 with the title 'Cara kerja dan daftar pestisida serta strategi pergilirannya pada budidaya tanaman sayuran dan palawija'. A lack of such type of standard agronomic information hampers the development of horticulture in Indonesia. Therefore, we decided to compile standard economic and agronomic information on a range of horticultural crops grown in Indonesia. It is based on information collected within vegIMPACT and its predecessor projects such as HORTIN I, HORTIN II and Train-the-Chain. The 'Handbook of vegetable production and economics in Indonesia' gives information on input prices, labour requirements, and quantified inputs and outputs of vegetable systems in terms of physical and economic units. This information is especially of interest for farmers, extension, researchers, project implementers, investors, financers, insurers and other stakeholders in horticulture. With the Handbook we contribute to making standard economic and agronomic horticultural information available and accessible to a wide range of stakeholders in Indonesia.

The Dutch Handbook Kwantitatieve Informatie voor de Akkerbouw en Vollegrondsgroenteteelt (Spruijt and Van der Voort, 2015) was an important source of inspiration for the present Handbook but the former is built on a long tradition of systematically collecting data on agriculture in the Netherlands that is yet lacking in Indonesia. Therefore, this Handbook only contains information on a limited number of vegetable crops and spatial and temporal information on input and output prices, crop inputs and outputs were only available to a limited extent. Therefore, this first edition of the Handbook of vegetable production and economics in Indonesia is also a call for more systematic data collection at a wider scale across the different islands of Indonesia.

The authors 


\section{Introduction}

\subsection{General introduction}

This Handbook compiles data on input prices, labour requirements, and inputs and outputs of a number of vegetable crops in physical and economic terms. Most data and information has been collected in the vegIMPACT program, which aims to improve vegetable production and marketing of small farmers in Indonesia (see: www.vegimpact.com). In addition, also suitable information that was collected in predecessor projects, i.e. HORTIN I and II, and Train the Chain, have been compiled, analysed and presented in this Handbook.

The systematically collected data and information on vegetable crops grown in Indonesia is scarce. This Handbook is a first effort to realize an independent reference guide on vegetable production in Indonesia. It is acknowledged that this Handbook with both economic and agronomic data on vegetable crops in Indonesia is yet based on a limited data set. However, systematically collected economic and agronomic data are the basis for more informed-decision making related to a range of sectors and stakeholders, including banking and finance, environment, business development, agronomic research, extension and farmers.

Indonesia with its many islands covers a huge land mass with different agro-ecological and socioeconomic conditions characterising local vegetable production. The total area with major vegetables (potato, chili, shallot, cabbage, carrot and onion) in Indonesia was about 570.000 ha in 2013 (PBS, 2014). More than $60 \%$ of this vegetable area was on Java. The vegIMPACT program and also its predecessor project mainly focused on Java. Therefore, most data and information presented in this Handbook refer to Java. But even Java is large and conditions are not the same across the island. In this Handbook information of highland and lowland vegetable crops is presented, and to further account for spatial and temporal variation in data, price series of variable inputs (fertilizers and crop protection agents) are presented when available, and for some vegetables the gross margins under wet season and dry season conditions are presented.

\subsection{Data and methods}

This handbook presents key agronomic and economic data on vegetable production in Indonesia. Use of these data requires understanding how they have been collected and calculated. The economic and agronomic data presented is collected by farmers, and therefore, the shown data do not represent a 'best practice', i.e. the minimum of each production resource that is needed to allow maximum utilization of all other resources (De Wit, 1992).

During the course of the vegIMPACT program agronomic data on potatoes and other vegetables was made available through the multi-season monitoring study, which focused on improving potato and vegetable production in Indonesia (Van den Brink et.al., 2015, Pronk et.al., 2017). This agronomic data was analysed to identify options for improvements in crop performance and resource use efficiencies. In addition to the agronomic assessment, an economic assessment was included. This assessment was carried out per individual crop/farmer. The economic assessment per crop/farmer was the starting point of this handbook. The regions, crops, seasons and other relevant data and detailed information are available in vegIMPACT reports (Van den Brink et.al., 2015, Pronk et.al., 2017, De Putter et.al., 2013, De Putter et.al., 2014). 
KWIN was a reference source for this Handbook (Spruijt and Van der Voort, 2015). The KWIN is made up out of two main parts, first general economic data and second crop gross margins. In this Handbook, Chapter 2 presents average economic data, such as prices of fertilizer, crop protection, labour requirements and land prices. The prices of most used fertilizers and crop protection agents in vegetables are available from above mentioned studies of the vegIMPACT program. Because the use of fertilizers and crop protection agents differs among farmers only the fertilizers and crop protection products used by farmers in multiple years are presented in Chapter 2. The used crop protection products are alphabetically ordered according to their active ingredient name. The prices are expressed per unit commercial product (gram, $\mathrm{ml}, \mathrm{l}$, etc.) in which they are sold at retail shops.

The labour requirements in crop production depend among others on the agro-ecological conditions (e.g. soil type, terrain characteristics, crop type and variety, planting density), skills and attitude of farmers and labourers, and on the local production situation (i.e. level of mechanization, input use) and associated yield level, which is not specified as the data are averages from different farmers. Hence, the presented data on labour requirements need to be used with care. The labour requirements of crops are specified per major field operation, i.e. land preparation, sowing/planting, fertilization, crop protection, crop maintenance, irrigation, weeding and harvest. Furthermore, we distinguish labour requirement as recorded by farmers according to gender because labour wages usually differ between men and women, which affects the financial cost-benefit calculations. Some operations are predominantly carried out by women, while others more by men.

The second part of this handbook are the gross margins of different crops for Indonesia. Chapter 3 presents the calculated gross margins of a number of crops. A gross margin of a crop is defined as the total average financial output minus the variable input costs, including the costs for hired labour. The output comprises the market value of the crop including the production retained the farm. The inputs and outputs of the gross margin calculations are further discussed and described in Chapter 3 . The inputs, outputs and gross margins of crops are expressed per hectare. The gross margin is not the same as profit, because no fixed costs and overhead costs are taken in account. The given average gross margins can be considered as a reference value, and do not represent the economic performance of an actual individual farmer. 


\section{Variable costs}

\subsection{Introduction}

To generate reference data for economic modelling and calculation on vegetable production in Indonesia the prices, characteristics and nutrient content of different fertilizer types, prices of crop protection agents and the labour requirements for different field operations are presented in this Chapter. The use of these inputs varies significantly among farmer and crop. These price data have also been used in the calculation of the gross margins. All prices are expressed in Indonesian Rupiah (IDR). These general data on variable costs is a starting point for the ex-ante economic evaluation of alternative production methods.

\subsection{Fertilization}

Following fertilizers are the most used fertilizer types in potato and other vegetables at Java, Indonesia. The shown price data are average prices based on observed prices in 2013 and 2014.

\begin{tabular}{|c|c|c|c|c|c|c|c|c|}
\hline \multirow{2}{*}{$\begin{array}{l}\text { Sort of } \\
\text { Fertilizer }\end{array}$} & \multirow[b]{2}{*}{ Name/Brand } & \multicolumn{3}{|l|}{ Price } & \multicolumn{3}{|c|}{ Contents } & \multirow[b]{2}{*}{ Other } \\
\hline & & IDR/unit & unit & Type & $\mathbf{N}$ & $\mathbf{P}$ & $\mathbf{K}$ & \\
\hline inorganic & NPK 15:15:15 & 3,200 & $\mathrm{~kg}$ & granular & 15 & 15 & 15 & Zn: $0.1 \%$ \\
\hline inorganic & $\mathrm{KCL}$ & 6,303 & $\mathrm{~kg}$ & granular & & & & \\
\hline inorganic & NPK 16:16:16 & 8,621 & $\mathrm{~kg}$ & granular & 16 & 16 & 16 & \\
\hline inorganic & Urea & 2,644 & $\mathrm{~kg}$ & granular & 46 & 0 & 0 & \\
\hline inorganic & SP 36 & 2,291 & $\mathrm{~kg}$ & granular & 0 & 36 & 0 & \\
\hline inorganic & ZA & 2,142 & $\mathrm{~kg}$ & & 21 & & & \\
\hline inorganic & Gandasil & 159,500 & $\mathrm{~kg}$ & foliar & 6 & 15 & 15 & MgSO4: 1\% \\
\hline inorganic & Grand k (13:0:46) & 26,183 & $\mathrm{~kg}$ & combi gran./fol. & 13 & 0 & 46 & \\
\hline inorganic & Kalsium & 16,500 & $\mathrm{~kg}$ & lime/soil improvement & & & & \\
\hline inorganic & DAP & 8,333 & $\mathrm{~kg}$ & granular & 18 & 46 & 0 & \\
\hline inorganic & Dolomit & 413 & $\mathrm{~kg}$ & lime/soil improvement & & & & $\mathrm{CaO}: \pm 30 \%$; MgO: $18-22 \%$ \\
\hline inorganic & Grower 15:9:20 & 9,370 & $\mathrm{~kg}$ & granular & 15 & 9 & 20 & \\
\hline inorganic & KNO & 10,011 & gram & granular & 13 & 0 & 46 & \\
\hline organic & Pupuk kandang & 482 & $\mathrm{~kg}$ & organic & & & & \\
\hline organic & $\begin{array}{l}\text { Pupuk kandang } \\
\text { ayam }\end{array}$ & 475 & $\mathrm{~kg}$ & organic & & & & \\
\hline inorganic & Saprodap & 7,478 & $\mathrm{~kg}$ & granular & 16 & 20 & 0 & \\
\hline inorganic & $\begin{array}{l}\text { Ammonium } \\
\text { sulphate }\end{array}$ & 3,773 & $\mathrm{~kg}$ & granular & 21 & 0 & 0 & SO4: $24 \%$ \\
\hline inorganic & Atonik & 330 & $\mathrm{ml}$ & foliar & & & & \\
\hline inorganic & Buril & 125 & gram & & & & & \\
\hline inorganic & Fertiphos 0:20:0 & 2,600 & $\mathrm{~kg}$ & granular & 0 & 20 & 0 & $\begin{array}{l}\mathrm{MgO}: 3 \% ; \mathrm{S}: 1 \% ; \mathrm{B} 2 \mathrm{O} 3: \\
0.2 \% \text {; } \mathrm{CaO}: 20 \%\end{array}$ \\
\hline
\end{tabular}




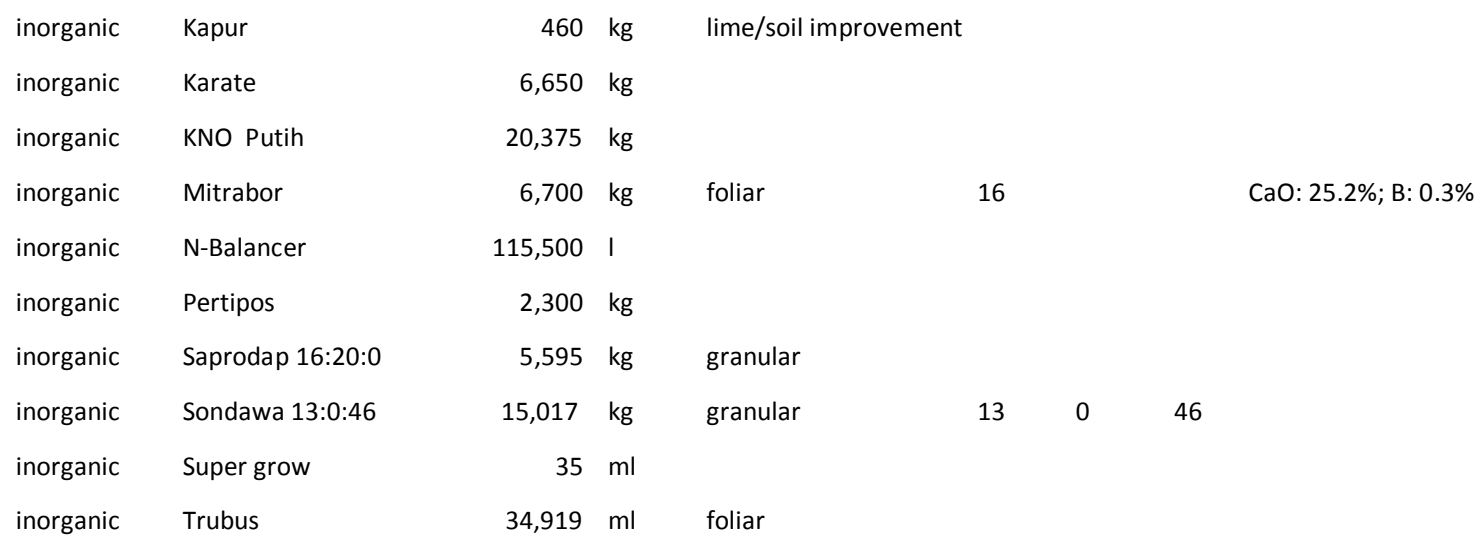




\subsection{Crop protection}

The price per crop protection agent is given in Indonesian Rupiah (IDR) per unit ( $\mathrm{ml}$ or grams). The data are not listed according brand names of crop production products but instead by the active ingredient of the products. However, aid materials are listed by their brand names.

\begin{tabular}{|c|c|c|c|}
\hline Active substance & Type $^{1}$ & IDR/unit & unit \\
\hline azoxystrobin & $\mathrm{F}$ & 666 & $\mathrm{ml}$ \\
\hline azoxystrobin + difenoconazole & $\mathrm{F}$ & 650 & $\mathrm{ml}$ \\
\hline chlorothalonil & $\mathrm{F}$ & 211 & gram \\
\hline cymoxanil & $\mathrm{F}$ & 411 & gram \\
\hline cymoxanil + famoxadone & $\mathrm{F}$ & 848 & gram \\
\hline difenoconazole & $\mathrm{F}$ & 519 & $\mathrm{ml}$ \\
\hline dimethomorph & $\mathrm{F}$ & 867 & gram \\
\hline iprodion & $\mathrm{F}$ & 459 & gram \\
\hline mancozeb & $\mathrm{F}$ & 88 & gram \\
\hline mancozeb + carbendazim & $\mathrm{F}$ & 146 & $\mathrm{ml}$ \\
\hline mancozeb + cymoxanil & $\mathrm{F}$ & 149 & gram \\
\hline mandipropamid & $\mathrm{F}$ & 713 & $\mathrm{ml}$ \\
\hline maneb & $\mathrm{F}$ & 60 & gram \\
\hline metalaxyl & $\mathrm{F}$ & 305 & $\mathrm{ml}$ \\
\hline metiram + pyraclostrobin & $\mathrm{F}$ & 177 & $\mathrm{ml}$ \\
\hline propamocarb hydrochloride & $\mathrm{F}$ & 269 & $\mathrm{ml}$ \\
\hline propiconazole & $\mathrm{F}$ & 170 & $\mathrm{ml}$ \\
\hline propineb & $\mathrm{F}$ & 119 & gram \\
\hline propineb + fluopicolide & $\mathrm{F}$ & 1,771 & gram \\
\hline pyraclostrobin & $\mathrm{F}$ & 888 & $\mathrm{ml}$ \\
\hline tebuconazole & $\mathrm{F}$ & 395 & $\mathrm{ml}$ \\
\hline thiophanate-methyl & $\mathrm{F}$ & 179 & $\mathrm{ml}$ \\
\hline trifloxystrobin + tebuconazole & $\mathrm{F}$ & 1,210 & gram \\
\hline glyphosate & $\mathrm{H}$ & 75 & $\mathrm{ml}$ \\
\hline isopropylamine glyphosate & $\mathrm{H}$ & 65 & $\mathrm{ml}$ \\
\hline
\end{tabular}

${ }^{1} \mathrm{~F}$-fungicide, $\mathrm{H}$-herbicide, I-Insecticide, $\mathrm{S}$-aid material 


\begin{tabular}{|c|c|c|}
\hline oxyfluorfen & $\mathrm{H}$ & $198 \mathrm{ml}$ \\
\hline paraquat & $\mathrm{H}$ & $887 \mathrm{ml}$ \\
\hline Paraquat dichloride & $\mathrm{H}$ & $99 \mathrm{ml}$ \\
\hline pendimethalin & $\mathrm{H}$ & $147 \mathrm{ml}$ \\
\hline abamectin & I & $797 \mathrm{ml}$ \\
\hline acetamiprid & I & $325 \mathrm{ml}$ \\
\hline alfa cypermethrin & I & $216 \mathrm{ml}$ \\
\hline beta-cyfluthrin & 1 & $163 \mathrm{ml}$ \\
\hline beta-cypermethrin & I & $130 \mathrm{ml}$ \\
\hline cadusafos & I & $43,500 \mathrm{ml}$ \\
\hline carbofuran & 1 & $10 \mathrm{~kg}$ \\
\hline Carbofuran & 1 & $17,403 \mathrm{~kg}$ \\
\hline carbosulfan & I & $162 \mathrm{ml}$ \\
\hline chlorantraniliprole & 1 & $705 \mathrm{ml}$ \\
\hline chlorfenapyr & 1 & $552 \mathrm{ml}$ \\
\hline chlorfluazuron & 1 & $645 \mathrm{ml}$ \\
\hline chlorpyrifos & 1 & $107 \mathrm{ml}$ \\
\hline chlorpyrifos + cypermethrin & 1 & $148 \mathrm{ml}$ \\
\hline cypermethrin & 1 & $167 \mathrm{ml}$ \\
\hline cyromazine & I & 2,580 gram \\
\hline deltamethrin & I & $420 \mathrm{ml}$ \\
\hline diafenthiuron & I & $2,124 \mathrm{ml}$ \\
\hline dimehypo & 1 & $78 \mathrm{ml}$ \\
\hline emamectin benzoate & I & $431 \mathrm{ml}$ \\
\hline fenobucarb & I & $107 \mathrm{ml}$ \\
\hline fipronil & I & $178 \mathrm{~kg}$ \\
\hline fipronil & 1 & $18,314 \mathrm{~kg}$ \\
\hline imidacloprid & I & $482 \mathrm{ml}$ \\
\hline lambda cyhalothrin & I & $309 \mathrm{ml}$ \\
\hline lufenuron & 1 & $319 \mathrm{ml}$ \\
\hline methamidophos & 1 & $210 \mathrm{ml}$ \\
\hline methomyl & I & 207 gram \\
\hline permethrin & 1 & $201 \mathrm{ml}$ \\
\hline
\end{tabular}




\begin{tabular}{|c|c|c|}
\hline profenofos & I & 214 \\
\hline spinetoram & I & 1,192 \\
\hline spiromesifen & I & 539 \\
\hline thiamethoxam & I & 1,545 \\
\hline thiamethoxam + lambda-cyhalothrin & । & 705 \\
\hline thiodicarb & I & 381 \\
\hline triazophos & I & 342 \\
\hline Apsa & S & 117 \\
\hline Balistic & S & 218 \\
\hline Besmor & S & 168 \\
\hline Borer & S & 170 \\
\hline Dustik & S & 53 \\
\hline Indostik & S & 7,345 \\
\hline Lantis & S & 37 \\
\hline Napel & S & 12 \\
\hline Perekat & $S$ & 40 \\
\hline Rohastik & $S$ & 35 \\
\hline Triple-X & $S$ & 30 \\
\hline Triton-X & $S$ & 106 \\
\hline
\end{tabular}




\subsection{Labour requirements}

The labour requirements of field operations in potato are shown per gender and for different years because several years of data were available. The subdivision according gender is important as wages of male and female field workers often differ, which affects the labour costs. In our data collection we observed average wages for men of IDR 8,000 per hour and for woman of IDR 4,600 per hour. The total share provides the distribution of labour carried out by male and female workers. In addition, the share per operation is given as percentage of the total labour. These shares per operations do not necessarily sum up to $100 \%$ because multiple data sources have been used.

The number of farmers used for calculating the labour requirement is the same as used for calculating the gross margins of crops (Chapter 3 ).

\section{Potatoes}

Labour requirement per hectare of potatoes - 2014

\begin{tabular}{|c|c|c|c|c|}
\hline Gender / Operation & & Hours & $\begin{array}{r}\text { Share male / } \\
\text { female }\end{array}$ & Share per operation \\
\hline \multirow[t]{9}{*}{ Male labour (total) } & & 1763 & $54 \%$ & \\
\hline & Land preparation & 431 & & $14 \%$ \\
\hline & Sowing/planting & 122 & & $4 \%$ \\
\hline & Fertilization & 200 & & $6 \%$ \\
\hline & Crop protection & 602 & & $19 \%$ \\
\hline & Crop maintenance & 196 & & $6 \%$ \\
\hline & Irrigation & & & \\
\hline & Weeding & 115 & & $4 \%$ \\
\hline & Harvest & 279 & & $9 \%$ \\
\hline \multirow[t]{9}{*}{ Female labour (total) } & & 1402 & $46 \%$ & \\
\hline & Land preparation & 505 & & $16 \%$ \\
\hline & Sowing/planting & 171 & & $5 \%$ \\
\hline & Fertilization & 236 & & $8 \%$ \\
\hline & Crop protection & 195 & & $6 \%$ \\
\hline & Crop maintenance & 239 & & $8 \%$ \\
\hline & Irrigation & & & \\
\hline & Weeding & 203 & & $6 \%$ \\
\hline & Harvest & 409 & & $13 \%$ \\
\hline
\end{tabular}

Total labour hours per hectare of potatoes 
Labour requirement per hectare of potatoes - 2015

\begin{tabular}{|c|c|c|c|}
\hline Gender / Operation & Hours & $\begin{array}{r}\text { Share male / } \\
\text { female }\end{array}$ & $\begin{array}{l}\text { Share per } \\
\text { operation }\end{array}$ \\
\hline Male labour (total) & 1897 & $59 \%$ & \\
\hline Land preparation & 414 & & $13 \%$ \\
\hline Sowing/planting & 144 & & $5 \%$ \\
\hline Fertilization & 240 & & $8 \%$ \\
\hline Crop protection & 588 & & $19 \%$ \\
\hline Crop maintenance & 368 & & $12 \%$ \\
\hline Irrigation & 0 & & $0 \%$ \\
\hline Weeding & 0 & & $0 \%$ \\
\hline Harvest & 246 & & $8 \%$ \\
\hline Female labour (total) & 1201 & $41 \%$ & \\
\hline Land preparation & 300 & & $9 \%$ \\
\hline Sowing/planting & 167 & & $6 \%$ \\
\hline Fertilization & 237 & & $7 \%$ \\
\hline Crop protection & 168 & & $5 \%$ \\
\hline Crop maintenance & 387 & & $13 \%$ \\
\hline Irrigation & 0 & & $0 \%$ \\
\hline Weeding & 0 & & $0 \%$ \\
\hline Harvest & 387 & & $13 \%$ \\
\hline
\end{tabular}

Total labour hours per

hectare of potatoes

3098 
Labour requirement per hectare of potatoes - 2016

\begin{tabular}{|c|c|c|c|}
\hline Gender / Operation & Hours & $\begin{array}{r}\text { Share male / } \\
\text { female }\end{array}$ & $\begin{array}{l}\text { Share per } \\
\text { operation }\end{array}$ \\
\hline Male labour (total) & 1305 & $44 \%$ & \\
\hline Land preparation & 271 & & $9 \%$ \\
\hline Sowing/planting & 119 & & $5 \%$ \\
\hline Fertilization & 144 & & $5 \%$ \\
\hline Crop protection & 476 & & $17 \%$ \\
\hline Crop maintenance & 246 & & $9 \%$ \\
\hline Irrigation & 0 & & $0 \%$ \\
\hline Weeding & 17 & & $1 \%$ \\
\hline Harvest & 256 & & $10 \%$ \\
\hline Female labour (total) & 1347 & $56 \%$ & \\
\hline Land preparation & 302 & & $10 \%$ \\
\hline Sowing/planting & 194 & & $8 \%$ \\
\hline Fertilization & 217 & & $9 \%$ \\
\hline Crop protection & 276 & & $14 \%$ \\
\hline Crop maintenance & 471 & & $17 \%$ \\
\hline Irrigation & 0 & & $0 \%$ \\
\hline Weeding & 0 & & $0 \%$ \\
\hline Harvest & 397 & & $16 \%$ \\
\hline
\end{tabular}

Total labour hours per hectare of potatoes 


\section{Other vegetable crops}

Labour requirement per hectare of shallot

\begin{tabular}{|c|c|c|c|}
\hline Gender / Operation & Hours & $\begin{array}{l}\text { Share male } \\
\text { / female }\end{array}$ & $\begin{array}{l}\text { Share per } \\
\text { operation }\end{array}$ \\
\hline Male labour (total) & 2273 & $60 \%$ & \\
\hline Land preparation & 704 & & $17 \%$ \\
\hline Sowing/planting & 58 & & $1 \%$ \\
\hline Fertilization & 102 & & $3 \%$ \\
\hline Crop protection & 473 & & $9 \%$ \\
\hline Crop maintenance & 149 & & $3 \%$ \\
\hline Irrigation & 701 & & $20 \%$ \\
\hline Weeding & 152 & & $3 \%$ \\
\hline Harvest & 249 & & $8 \%$ \\
\hline Female labour (total) & 1571 & $40 \%$ & \\
\hline Land preparation & 290 & & $7 \%$ \\
\hline Sowing/planting & 417 & & $12 \%$ \\
\hline Fertilization & 169 & & $6 \%$ \\
\hline Crop protection & 334 & & $9 \%$ \\
\hline Crop maintenance & 217 & & $9 \%$ \\
\hline Irrigation & 73 & & $2 \%$ \\
\hline Weeding & 479 & & $12 \%$ \\
\hline Harvest & 391 & & $11 \%$ \\
\hline
\end{tabular}

Total Labour hours per hectare

3845

vegIMPACT Report 48 - Handbook of vegetable production and economics in Indonesia 
Labour requirement per hectare of sweet corn

\begin{tabular}{|c|c|c|c|}
\hline Gender / Operation & Hours & $\begin{array}{r}\text { Share male / } \\
\text { female }\end{array}$ & $\begin{array}{l}\text { Share per } \\
\text { operation }\end{array}$ \\
\hline Male labour (total) & 519 & $60 \%$ & \\
\hline Land preparation & 158 & & $30 \%$ \\
\hline Sowing/planting & 45 & & $9 \%$ \\
\hline Fertilization & 50 & & $10 \%$ \\
\hline Crop protection & 126 & & $24 \%$ \\
\hline Crop maintenance & 0 & & $0 \%$ \\
\hline Irrigation & 95 & & $18 \%$ \\
\hline Weeding & 37 & & $7 \%$ \\
\hline Harvest & 82 & & $16 \%$ \\
\hline Female labour (total) & 351 & $40 \%$ & \\
\hline Land preparation & 54 & & $15 \%$ \\
\hline Sowing/planting & 83 & & $24 \%$ \\
\hline Fertilization & 137 & & $39 \%$ \\
\hline Crop protection & 85 & & $24 \%$ \\
\hline Crop maintenance & 0 & & $0 \%$ \\
\hline Irrigation & 0 & & $0 \%$ \\
\hline Weeding & 150 & & $43 \%$ \\
\hline Harvest & 59 & & $17 \%$ \\
\hline
\end{tabular}

Total Labour hours per hectare 
Labour requirement per hectare of water spinach

\begin{tabular}{|c|c|c|c|}
\hline Gender / Operation & Hours & $\begin{array}{r}\text { Share male / } \\
\text { female }\end{array}$ & $\begin{array}{l}\text { Share pe } \\
\text { operation }\end{array}$ \\
\hline Male labour (total) & 1,850 & $35 \%$ & \\
\hline Land preparation & 400 & & $22 \%$ \\
\hline Sowing/planting & 100 & & $5 \%$ \\
\hline Fertilization & 350 & & $19 \%$ \\
\hline Crop protection & 100 & & $5 \%$ \\
\hline Crop maintenance & 0 & & $0 \%$ \\
\hline Irrigation & 0 & & $0 \%$ \\
\hline Weeding & 0 & & $0 \%$ \\
\hline Harvest & 1,200 & & $65 \%$ \\
\hline Female labour (total) & 3,500 & $65 \%$ & \\
\hline Land preparation & 400 & & $11 \%$ \\
\hline Sowing/planting & 350 & & $10 \%$ \\
\hline Fertilization & 350 & & $10 \%$ \\
\hline Crop protection & 0 & & $0 \%$ \\
\hline Crop maintenance & 0 & & $0 \%$ \\
\hline Irrigation & 0 & & $0 \%$ \\
\hline Weeding & 1,400 & & $40 \%$ \\
\hline Harvest & 1,200 & & $34 \%$ \\
\hline
\end{tabular}

Total Labour hours per hectare

5,350 
Labour requirement per hectare of rice

\begin{tabular}{|c|c|c|c|}
\hline Gender / Operation & Hours & $\begin{array}{r}\text { Share male / } \\
\text { female }\end{array}$ & $\begin{array}{l}\text { Share per } \\
\text { operation }\end{array}$ \\
\hline Male labour (total) & 918 & $44 \%$ & \\
\hline Land preparation & 214 & & $23 \%$ \\
\hline Sowing/planting & 107 & & $12 \%$ \\
\hline Fertilization & 11 & & $1 \%$ \\
\hline Crop protection & 44 & & $5 \%$ \\
\hline Crop maintenance & 0 & & $0 \%$ \\
\hline Irrigation & 0 & & $0 \%$ \\
\hline Weeding & 800 & & $87 \%$ \\
\hline Harvest & 276 & & $30 \%$ \\
\hline Female labour (total) & 1,153 & $56 \%$ & \\
\hline Land preparation & 0 & & $0 \%$ \\
\hline Sowing/planting & 311 & & $27 \%$ \\
\hline Fertilization & 0 & & $0 \%$ \\
\hline Crop protection & 0 & & $0 \%$ \\
\hline Crop maintenance & 0 & & $0 \%$ \\
\hline Irrigation & 0 & & $0 \%$ \\
\hline Weeding & 842 & & $73 \%$ \\
\hline Harvest & 0 & & $0 \%$ \\
\hline
\end{tabular}

Total Labour hours per hectare

2,071 
Labour requirement per hectare of yard long bean

\begin{tabular}{|c|c|c|c|}
\hline Gender / Operation & Hours & $\begin{array}{r}\text { Share male / } \\
\text { female }\end{array}$ & $\begin{array}{l}\text { Share per } \\
\text { operation }\end{array}$ \\
\hline Male labour (total) & 1,541 & $48 \%$ & \\
\hline Land preparation & 28 & & $2 \%$ \\
\hline Sowing/planting & 28 & & $2 \%$ \\
\hline Fertilization & 48 & & $3 \%$ \\
\hline Crop protection & 263 & & $17 \%$ \\
\hline Crop maintenance & 259 & & $17 \%$ \\
\hline Irrigation & 643 & & $42 \%$ \\
\hline Weeding & 0 & & $0 \%$ \\
\hline Harvest & 618 & & $40 \%$ \\
\hline Female labour (total) & 1,696 & $52 \%$ & \\
\hline Land preparation & 106 & & $6 \%$ \\
\hline Sowing/planting & 106 & & $6 \%$ \\
\hline Fertilization & 48 & & $3 \%$ \\
\hline Crop protection & 0 & & $0 \%$ \\
\hline Crop maintenance & 0 & & $0 \%$ \\
\hline Irrigation & 0 & & $0 \%$ \\
\hline Weeding & 0 & & $0 \%$ \\
\hline Harvest & 1,459 & & $86 \%$ \\
\hline
\end{tabular}

Total Labour hours per hectare

3,237 
Labour requirement per hectare of hot pepper

\begin{tabular}{|c|c|c|c|}
\hline Gender / Operation & Hours & $\begin{array}{r}\text { Share male / } \\
\text { female }\end{array}$ & $\begin{array}{l}\text { Share per } \\
\text { operation }\end{array}$ \\
\hline Male labour (total) & 3,367 & $65 \%$ & \\
\hline Land preparation & 143 & & $4 \%$ \\
\hline Sowing/planting & 0 & & $0 \%$ \\
\hline Fertilization & 24 & & $1 \%$ \\
\hline Crop protection & 162 & & $5 \%$ \\
\hline Crop maintenance & 0 & & $0 \%$ \\
\hline Irrigation & 2,729 & & $81 \%$ \\
\hline Weeding & 0 & & $0 \%$ \\
\hline Harvest & 238 & & $7 \%$ \\
\hline Female labour (total) & 1,833 & $35 \%$ & \\
\hline Land preparation & 0 & & $0 \%$ \\
\hline Sowing/planting & 143 & & $8 \%$ \\
\hline Fertilization & 24 & & $1 \%$ \\
\hline Crop protection & 0 & & $0 \%$ \\
\hline Crop maintenance & 0 & & $0 \%$ \\
\hline Irrigation & 0 & & $0 \%$ \\
\hline Weeding & 238 & & $13 \%$ \\
\hline Harvest & 1,429 & & $78 \%$ \\
\hline
\end{tabular}

Total Labour hours per hectare $\quad 5,200$ 
Labour requirement per hectare of bittergourd

\begin{tabular}{|c|c|c|c|}
\hline Gender / Operation & Hours & $\begin{array}{r}\text { Share male / } \\
\text { female }\end{array}$ & $\begin{array}{l}\text { Share per } \\
\text { operation }\end{array}$ \\
\hline Male labour (total) & 1,286 & $64 \%$ & \\
\hline Land preparation & 48 & & $4 \%$ \\
\hline Sowing/planting & 24 & & $2 \%$ \\
\hline Fertilization & 48 & & $4 \%$ \\
\hline Crop protection & 190 & & $15 \%$ \\
\hline Crop maintenance & 119 & & $9 \%$ \\
\hline Irrigation & 738 & & $57 \%$ \\
\hline Weeding & 0 & & $0 \%$ \\
\hline Harvest & 119 & & $9 \%$ \\
\hline Female labour (total) & 714 & $36 \%$ & \\
\hline Land preperation & 0 & & $0 \%$ \\
\hline Sowing/planting & 24 & & $3 \%$ \\
\hline Fertilization & 48 & & $7 \%$ \\
\hline Crop protection & 190 & & $27 \%$ \\
\hline Crop maintenance & 333 & & $47 \%$ \\
\hline Irrigation & 0 & & $0 \%$ \\
\hline Weeding & 0 & & $0 \%$ \\
\hline Harvest & 119 & & $17 \%$ \\
\hline
\end{tabular}

Total Labour hours per hectare $\quad 2,000$ 


\subsection{Land prices}

The land prices are based on vegIMPACT surveys near Brebes, Cirebon and Pekalongan in 2016 (Witono Adiyoga and Herman de Putter, 2017) and the Train the Chain project in the Brebes region in 2010. All regions are on Java.

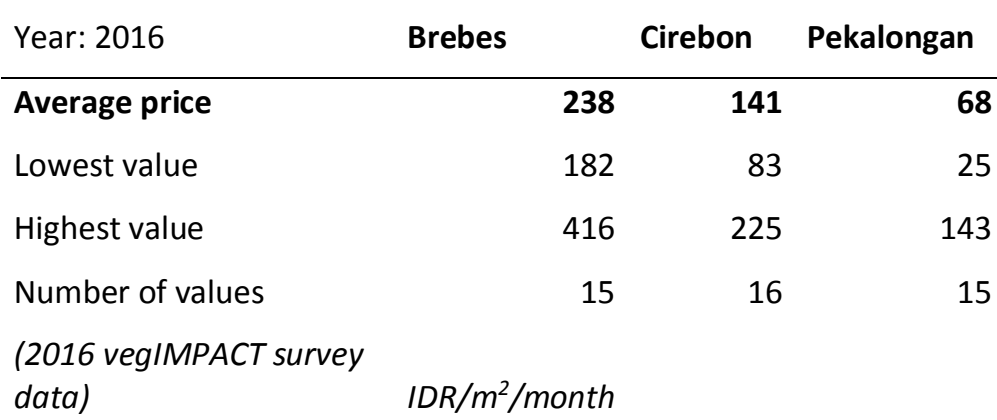

\begin{tabular}{lr} 
Year: 2010 & Brebes \\
\hline Average & 112 \\
Lowest value & 78 \\
Highest Value & 156 \\
Number of values & 8 \\
(2010 Train the Chain data) & $I D R / \mathrm{m}^{2} /$ month
\end{tabular}




\section{Gross margins}

\subsection{Principles of the gross margin calculations}

\section{General}

The gross margins of crops in this Handbook are based registered farmers' data within the vegIMPACT program (e.g. Van den Brink et.al., 2015, Pronk et.al., 2017, De Putter et.al., 2013, De Putter et.al., 2014). The number of farmers differs per crop, which is indicated with ( $n$ ) at the top of each gross margin calculation. The gross margins are expressed per hectare. The average field size is in most cases less than one hectare. The averages field size on which the gross margin is based indicated at the top of each calculation.

The gross margin is an average value, but varies considerably in practice. In order to provide an indication of this variation the median, lowest and highest values are provided.

The share of input costs in the total production costs is provided to indicate the importance of input costs in the total production costs.

In addition, the costs per kilogram product is calculated based on the yield and the total variable costs. This cost price provides an indication of the market price needed to cover all costs.

The production factors and crop income which determine the crop gross margin are described in detail in the following (all prices are in Indonesian Rupiah).

\section{Crop income (1.)}

Crop income is a combination of the physical/harvestable yield and the price of the products sold.

Specific for potatoes is that the yield is graded into different qualities/classes/grades. The most common marketable grades are A, B and C, each with a different price. Other grades include seed potatoes, damaged and rotten potatoes. The share of the total of grade $A, B$ and $C$ yield are expressed as a percentage of the total yield.

Specific for shallot is that the yield is based on a smaller number of farmers than the total income: Various farmers sell their 'field' and not the amount of product to a trader. Hence, these farmers have a crop income, but the actual yield is not known. This is expressed in the number of records used for yield.

\section{Seed / rootstock (2.)}

For all crops the amount of seed or rootstock used is provided, including the total costs.

\section{Fertilization (3.)}

The recorded data on fertilization showed a wide variety of fertilizer products with different prices used by farmers. Therefore, the fertilization costs are calculated as an average input cost. To provide agronomic insight in the fertilization per crop, the total amount of $\mathrm{N}$ (nitrogen), $\mathrm{P}_{2} \mathrm{O}_{5}$ (phosphate) and $\mathrm{K}_{2} \mathrm{O}$ (potassium) has been calculated based on the nutrient content of the used fertilizers and the amount applied per crop.

\section{Crop protection (4.)}

Similar to fertilization, the number of crop protection products used is large and diverse. For crop protection a similar approach is used as for fertilizers. The costs of fungicides, herbicides, insecticides and other crop protection agents are provided. Because crop protection agents can have different forms (solid and liquid forms) the amount of crop agents can vary depending on the formulation of the product. The other group crop protection includes, for example, adjuvants to improve operation and 
effect of the applied crop protection agent.

\section{Energy (5.)}

The energy costs include the costs for gasoline for motorised knapsack sprayers and vehicles to transport produce, fertilizer, manure, etc.

\section{Other materials and substances (6.)}

All other production materials and substances used are included. The main materials are bamboo sticks, rope and mulch.

\section{Labour requirements (7.)}

The labour requirements and costs are based on all field operations in a given crop. The labour requirements include the labour provided by the farmer and him/her family and hired labour. The amount of hired labour differs per crop area and is primarily dependent on farm size. Bigger farms have more hired labour. Therefore, the amount of labour and the labour costs are not comparable. Labour requirements for post-harvest operations are not included.

Contracted labour is not taken into account. This applies for example for ridging, which is done for multiple successive crops.

\section{Total variable costs (8.)}

The total variable costs are the sum of the costs for the production factors 2 to 8 .

\section{Gross margin (9.)}

The gross margin is calculated based on the average total income minus the total variable costs. 


\subsection{Potatoes}

Ware potatoes, Atlantic, wet season, per hectare

$\mathrm{n}=$ 28

Average field size: 0.37 ha

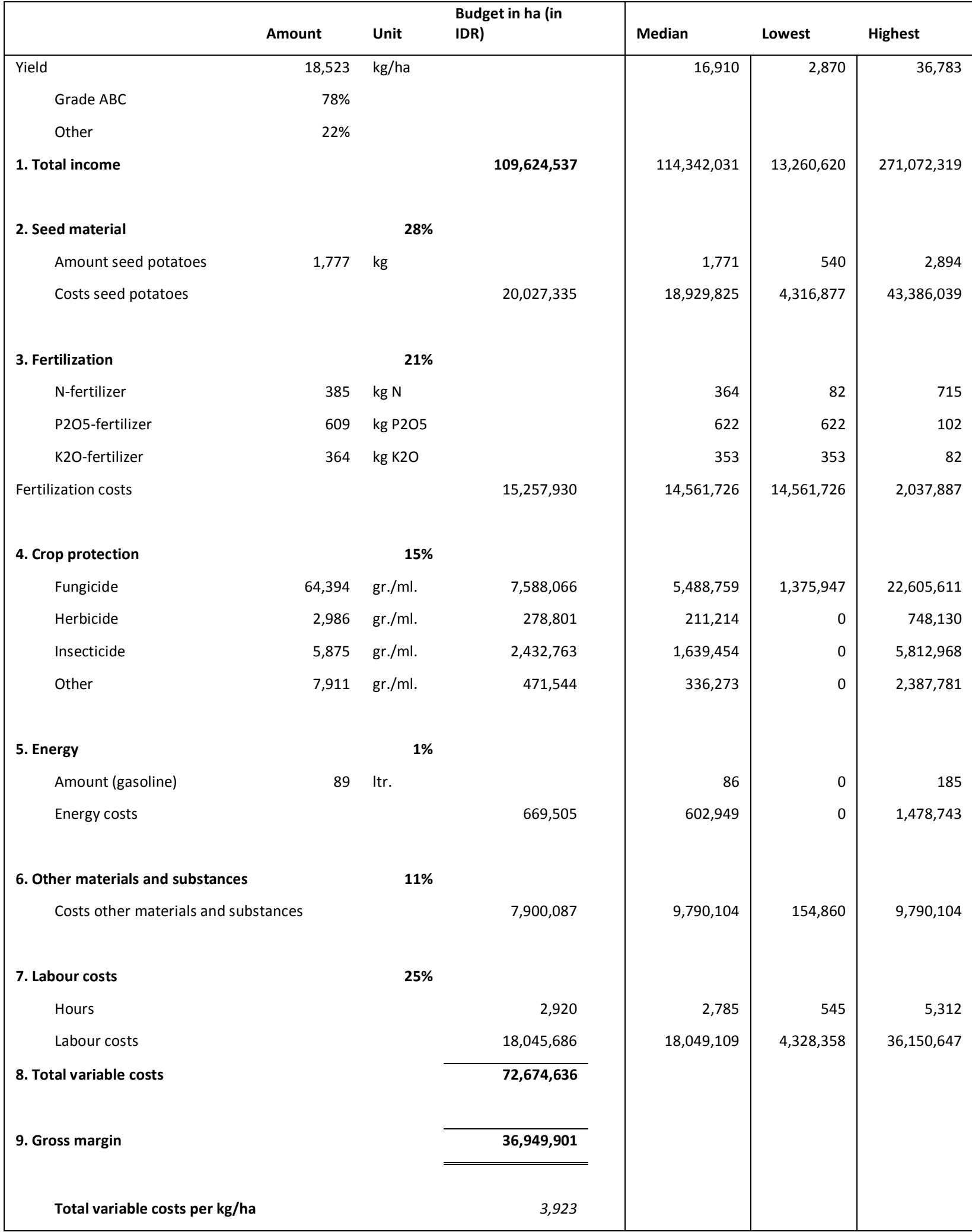


Ware potatoes, Atlantic, dry season, per hectare

$n=$ 9

Average field size:

0.39 ha

\begin{tabular}{|c|c|c|c|c|c|c|}
\hline $\mathrm{Am}$ & & Unit & $\begin{array}{l}\text { Budget in ha } \\
\text { (in IDR) }\end{array}$ & Median & Lowest & Highest \\
\hline Yield & 20,197 & $\mathrm{~kg} / \mathrm{ha}$ & & 18,042 & 12,879 & 34,251 \\
\hline Grade $A B C$ & $86 \%$ & & & & & \\
\hline Other & $14 \%$ & & & & & \\
\hline 1. Total income & & & $113,193,631$ & $104,301,075$ & $71,250,000$ & $179,210,918$ \\
\hline 2. Seed material & & $30 \%$ & & & & \\
\hline Amount seed potatoes & 1,867 & $\mathrm{~kg}$ & & 1,735 & 909 & 3,102 \\
\hline Costs seed potatoes & & & $23,343,706$ & $21,691,347$ & $11,363,636$ & $38,771,712$ \\
\hline 3. Fertilization & & $20 \%$ & & & & \\
\hline $\mathrm{N}$-fertilizer & 442 & $\mathrm{~kg} \mathrm{~N}$ & & 378 & 203 & 914 \\
\hline P2O5-fertilizer & 601 & kg P2O5 & & 520 & 236 & 924 \\
\hline K2O-fertilizer & 389 & kg K2O & & 378 & 203 & 567 \\
\hline Fertilization costs & & & $16,146,188$ & $14,328,949$ & $9,230,769$ & $23,490,488$ \\
\hline 4. Crop protection & & $13 \%$ & & & & \\
\hline Fungicide & 63,313 & gr./ml. & $7,315,723$ & $9,182,051$ & $2,137,960$ & $11,792,804$ \\
\hline Herbicide & 7,634 & $\mathrm{gr} . / \mathrm{ml}$. & 470,862 & 470,862 & 0 & 487,179 \\
\hline Insecticide & 4,261 & $\mathrm{gr} . / \mathrm{ml}$. & $2,058,451$ & $1,868,234$ & 613,421 & $4,164,599$ \\
\hline Other & 3,968 & $\mathrm{gr} . / \mathrm{ml}$. & 368,519 & 286,592 & 0 & 830,769 \\
\hline 5. Energy & & $1 \%$ & & & & \\
\hline Amount (gasoline) & 97 & Itr & & 92 & 0 & 170 \\
\hline Energy costs & & & 688,452 & 668,947 & 0 & $1,186,931$ \\
\hline 6. Other materials and substances & & $11 \%$ & & & & \\
\hline Costs other materials and substance & & & $8,390,993$ & $8,844,139$ & $3,136,364$ & $8,844,139$ \\
\hline 7. Labour costs & & $26 \%$ & & & & \\
\hline Labour requirement & 2,880 & hours & & 3,045 & 1,179 & 5,125 \\
\hline Labour costs & & & $20,182,818$ & $19,933,651$ & $13,635,241$ & $27,150,538$ \\
\hline 8. Total variable costs & & & $78,965,714$ & & & \\
\hline \multirow[t]{2}{*}{ 9. Gross margin } & & & $34,227,917$ & & & \\
\hline & I variable & costs per kg/ha & 3,910 & & & \\
\hline
\end{tabular}


Ware potatoes, Granola, wet season, per hectare

$\mathrm{n}=$ 47

Average field size: 0.22 ha

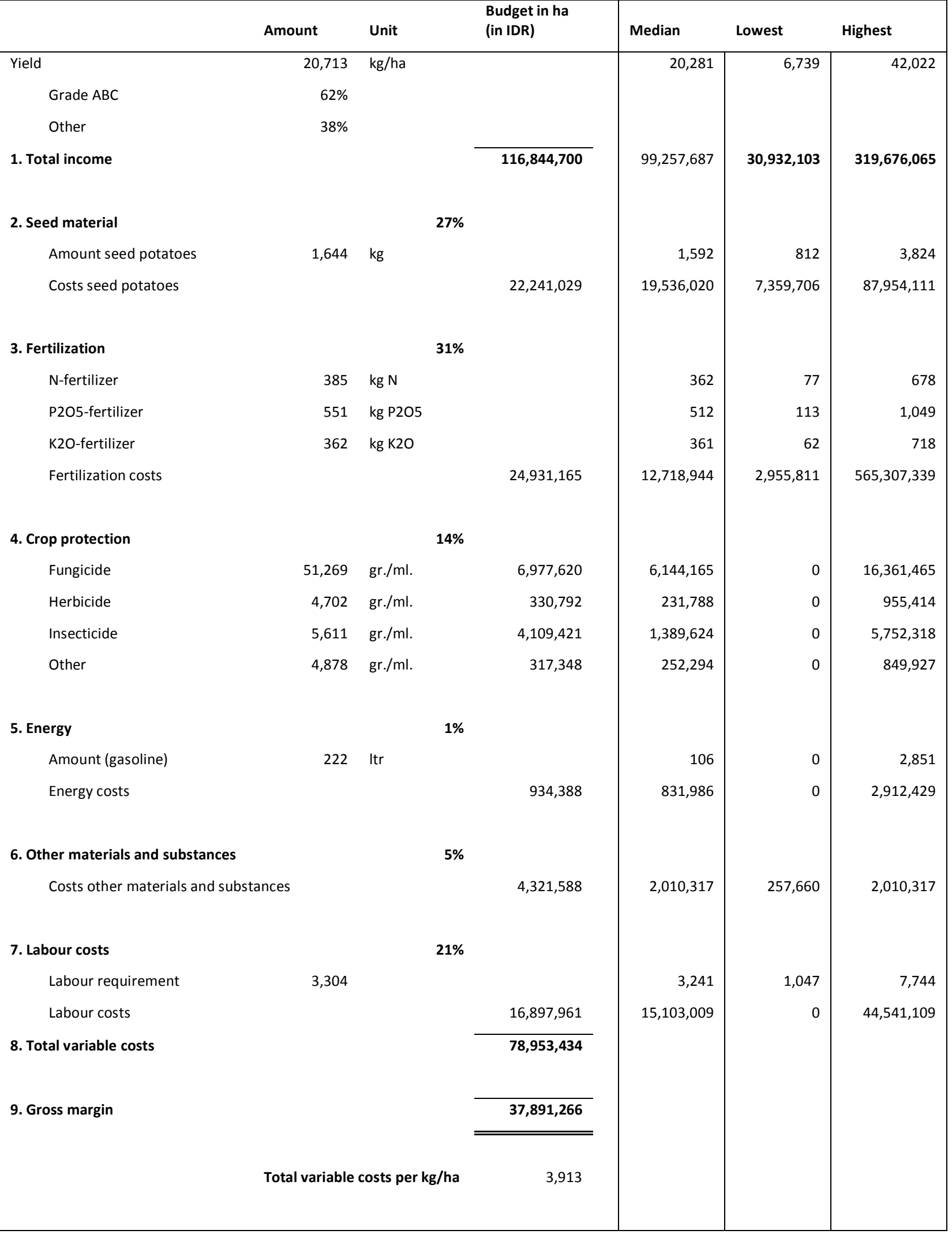


Ware potatoes, Granola, dry season, per hectare

$\mathrm{n}=$ 28

Average field size:

0.23 ha

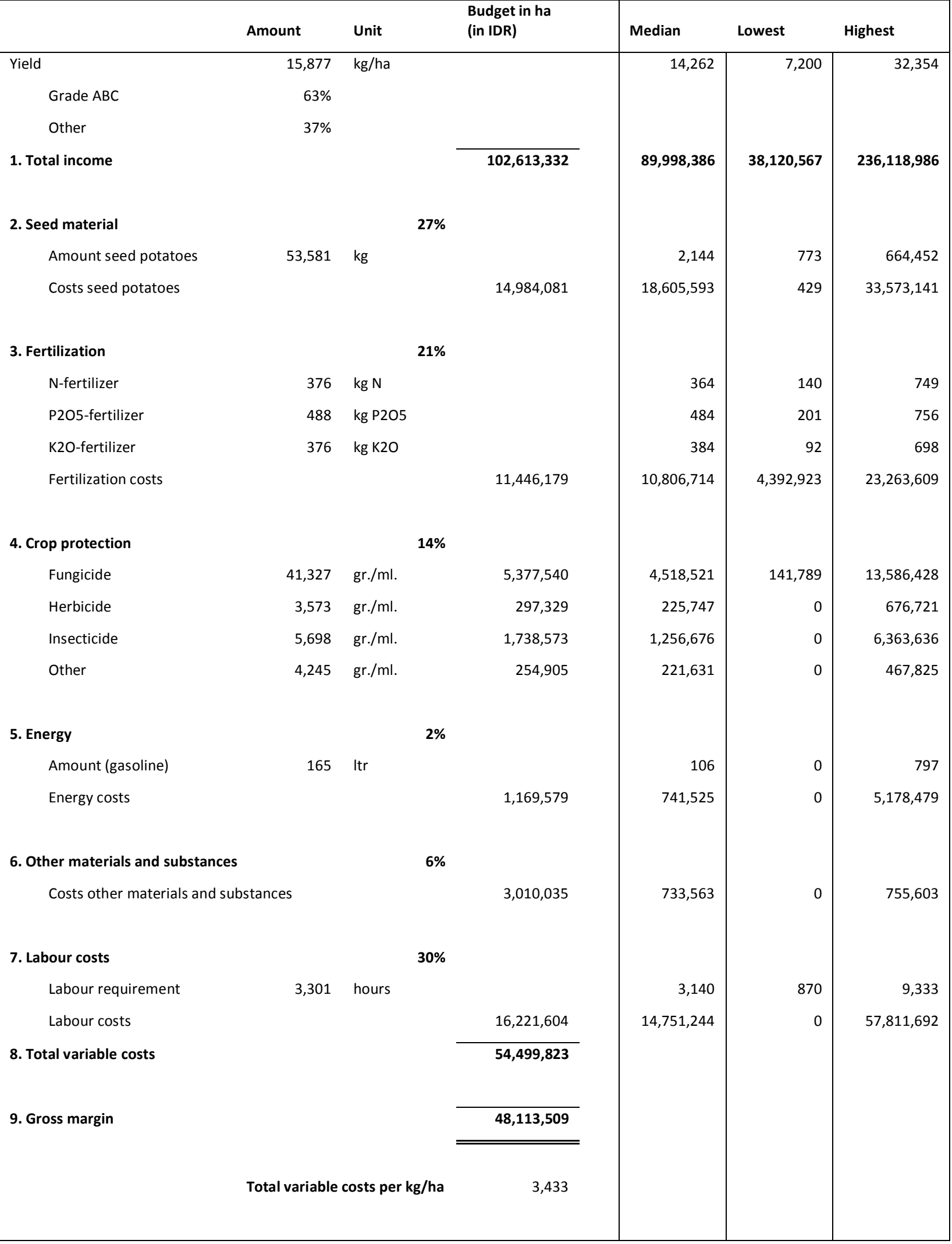




\subsection{Vegetable crops}

\section{Shallot, wet and dry season, per hectare}

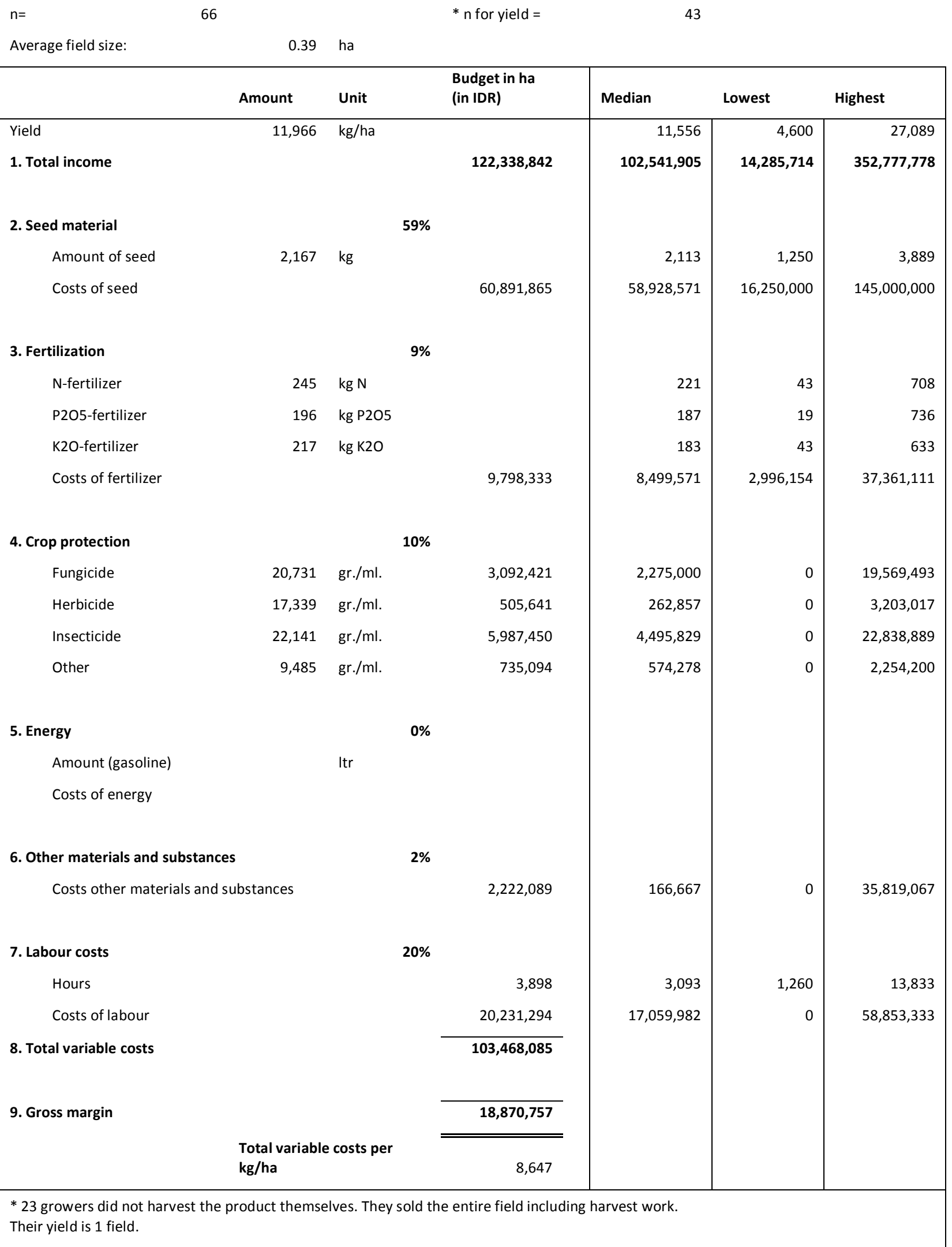




\section{Sweet corn, wet and dry season, average per hectare}

$\mathrm{n}=$

13

Average field size:

0.53 ha

\begin{tabular}{|c|c|c|c|c|c|c|}
\hline & Amount & Unit & $\begin{array}{l}\text { Budget in ha (in } \\
\text { IDR) }\end{array}$ & Median & Lowest & Highest \\
\hline Yield & 26,300 & $\mathrm{~kg} / \mathrm{ha}$ & & 19,643 & 8,304 & 64,286 \\
\hline 1. Total income & & & $47,413,379$ & $27,500,000$ & $15,446,429$ & $128,571,429$ \\
\hline 2. Seed material & & & & & & \\
\hline Amount of seed & 19,126 & $\mathrm{~kg}$ & & 13,839 & 0 & 48,214 \\
\hline Costs of seed & & & $3,579,762$ & $3,435,714$ & 0 & $7,142,857$ \\
\hline 3. Fertilization & & & & & & \\
\hline $\mathrm{N}$-fertilizer & 424 & $\mathrm{~kg} \mathrm{~N}$ & & 386 & 139 & 777 \\
\hline P2O5-fertilizer & 103 & kg P2O5 & & 74 & 11 & 321 \\
\hline K2O-fertilizer & 139 & kg K2O & & 129 & 13 & 441 \\
\hline Costs of fertilizer & & & $7,096,772$ & $3,665,625$ & $1,846,429$ & $35,098,214$ \\
\hline 4. Crop protection & & & & & & \\
\hline Fungicide & 5,466 & gr./ml. & $2,908,029$ & $1,010,714$ & 296,400 & $21,116,071$ \\
\hline Herbicide & 11,860 & gr./ml. & 541,214 & 461,929 & 0 & $1,269,286$ \\
\hline Insecticide & 5,128 & gr./ml. & $3,124,960$ & $1,962,857$ & 222,857 & $12,376,429$ \\
\hline Other & 3,123 & gr./ml. & 170,100 & 163,314 & 0 & 341,518 \\
\hline 5. Energy & & & & & & \\
\hline Amount (gasoline) & 165 & Itr & & 179 & 0 & 286 \\
\hline Costs of energy & & & $1,142,857$ & $1,225,000$ & 0 & $2,000,000$ \\
\hline 6. Other materials and sub & & & & & & \\
\hline Costs other materia & ubstances & & 158,571 & 128,571 & 0 & 321,429 \\
\hline 7. Labour costs & & & & & & \\
\hline Hours & & & 1,349 & 1,163 & 0 & 2,893 \\
\hline Costs of labour & & & $9,664,890$ & $7,767,857$ & $4,660,714$ & $22,285,714$ \\
\hline 8. Total variable costs & & & $28,388,505$ & & & \\
\hline \multirow{2}{*}{ 9. Gross margin } & & & $19,024,874$ & & & \\
\hline & \multicolumn{2}{|c|}{$\begin{array}{l}\text { Total variable costs per } \\
\mathrm{kg} / \mathrm{ha}\end{array}$} & 1,079 & & & \\
\hline
\end{tabular}


Water spinach, dry season, average per hectare

$\mathrm{n}=2$

Average field size: $\quad 0.05$ ha

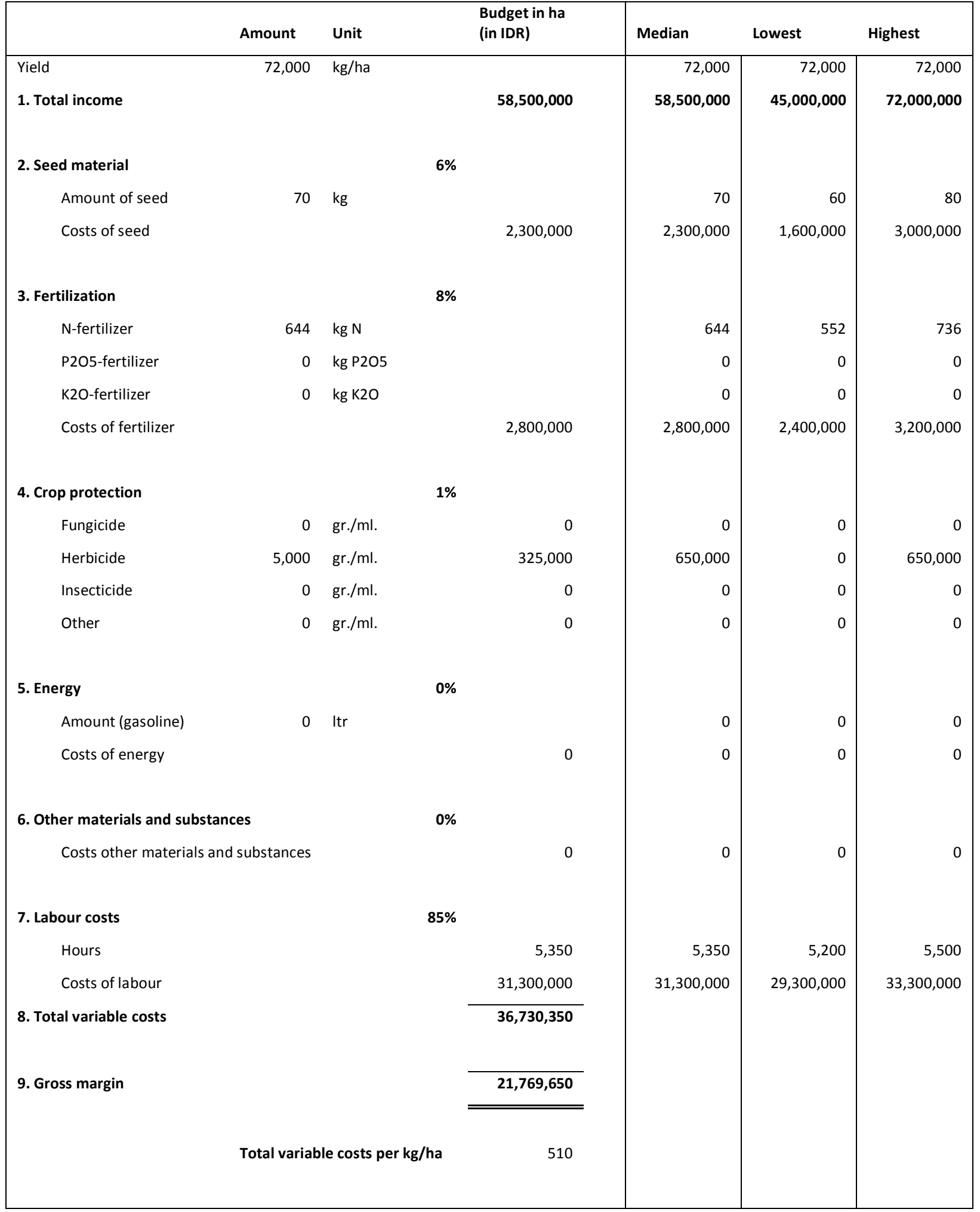


Yard Long Bean, wet and dry season, average per hectare

$n=$

2

Average field size:

0.19 ha

\begin{tabular}{|c|c|c|c|c|c|c|}
\hline & Amount & Unit & $\begin{array}{l}\text { Budget in ha } \\
\text { (in IDR) }\end{array}$ & Median & Lowest & Highest \\
\hline Yield & 15,594 & $\mathrm{~kg} / \mathrm{ha}$ & & 15,594 & 14,176 & 17,013 \\
\hline 1. Total income & & & $33,082,247$ & $33,082,247$ & $19,757,619$ & $46,406,875$ \\
\hline 2. Seed material & & $2 \%$ & & & & \\
\hline Amount of seed & 6 & $\mathrm{~kg}$ & & 6 & 5 & 6 \\
\hline Costs of seed & & & 550,595 & 550,595 & 476,190 & 625,000 \\
\hline 3. Fertilization & & $4 \%$ & & & & \\
\hline $\mathrm{N}$-fertilizer & 63 & $\mathrm{~kg} \mathrm{~N}$ & & 63 & 0 & 125 \\
\hline P2O5-fertilizer & 15 & kg P2O5 & & 15 & 0 & 29 \\
\hline K2O-fertilizer & 6 & kg K2O & & 6 & 0 & 12 \\
\hline Costs of fertilizer & & & 861,905 & 861,905 & 0 & $1,723,810$ \\
\hline 4. Crop protection & & $50 \%$ & & & & \\
\hline Fungicide & 5,536 & gr./ml. & $5,847,143$ & $5,847,143$ & $4,754,286$ & $6,940,000$ \\
\hline Herbicide & 0 & gr./ml. & 0 & 0 & 0 & 0 \\
\hline Insecticide & 5,143 & gr./ml. & $6,235,179$ & $6,235,179$ & $5,262,857$ & $7,207,500$ \\
\hline Other & 2,202 & gr./ml. & 55,060 & 55,060 & 47,619 & 62,500 \\
\hline 5. Energy & & $0 \%$ & & & & \\
\hline Amount (gasoline) & 0 & Itr & & 0 & 0 & 0 \\
\hline Costs of energy & & & 0 & 0 & 0 & 0 \\
\hline 6. Other materials and sub & & $0 \%$ & & & & \\
\hline Costs other materia & I substances & & 55,060 & 55,060 & 47,619 & 62,500 \\
\hline 7. Labour costs & & $44 \%$ & & & & \\
\hline Hours & & & 3,237 & 3,237 & 3,188 & 3,286 \\
\hline Costs of labour & & & $10,821,429$ & $10,821,429$ & 0 & $21,642,857$ \\
\hline 8. Total variable costs & & & $24,429,606$ & & & \\
\hline \multirow[t]{2}{*}{ 9. Gross margin } & & & $8,652,641$ & & & \\
\hline & \multicolumn{2}{|c|}{ Total variable costs per kg/ha } & 1,567 & & & \\
\hline
\end{tabular}




\section{Hot pepper, wet season, average per hectare}

$\mathrm{n}=$

1

Average field size: $\quad 0.21$ ha

\begin{tabular}{|c|c|c|c|}
\hline & punt & Unit & $\begin{array}{l}\text { Budget in ha (in } \\
\text { IDR) }\end{array}$ \\
\hline Yield & 7,090 & $\mathrm{~kg} / \mathrm{ha}$ & \\
\hline 1. Total income & & & $35,004,762$ \\
\hline 2. Seed material & & $1 \%$ & \\
\hline Amount of seed & 1,429 & $\mathrm{~kg}$ & \\
\hline Costs of seed & & & 428,571 \\
\hline 3. Fertilization & & $5 \%$ & \\
\hline $\mathrm{N}$-fertilizer & 238 & $\mathrm{~kg} \mathrm{~N}$ & \\
\hline P2O5-fertilizer & 41 & kg P2O5 & \\
\hline K2O-fertilizer & 11 & kg K2O & \\
\hline Costs of fertilizer & & & $2,602,381$ \\
\hline 4. Crop protection & & $16 \%$ & \\
\hline Fungicide & 8,000 & gr./ml. & 832,000 \\
\hline Herbicide & 4,785 & gr./ml. & 118,362 \\
\hline Insecticide & 9,143 & gr./ml. & $6,646,857$ \\
\hline Other & 5,333 & gr./ml. & 533,333 \\
\hline 5. Energy & & $6 \%$ & \\
\hline Amount (gasoline) & 448 & Itr & \\
\hline Costs of energy & & & $3,133,333$ \\
\hline 6. Other materials and substances & & $0 \%$ & \\
\hline Costs other materials and su & stances & & 0 \\
\hline 7. Labour costs & & $72 \%$ & \\
\hline Hours & & & 5,200 \\
\hline Costs of labour & & & $36,100,000$ \\
\hline 8. Total variable costs & & & $50,400,038$ \\
\hline \multirow[t]{2}{*}{ 9. Gross margin } & & & $-15,395,276$ \\
\hline & \multicolumn{2}{|c|}{ Total variable costs per kg/ha } & 7,108 \\
\hline
\end{tabular}


Bitter-gourd, wet season, average per hectare

$\mathrm{n}=$

Average field size: $\quad 0.21$ ha

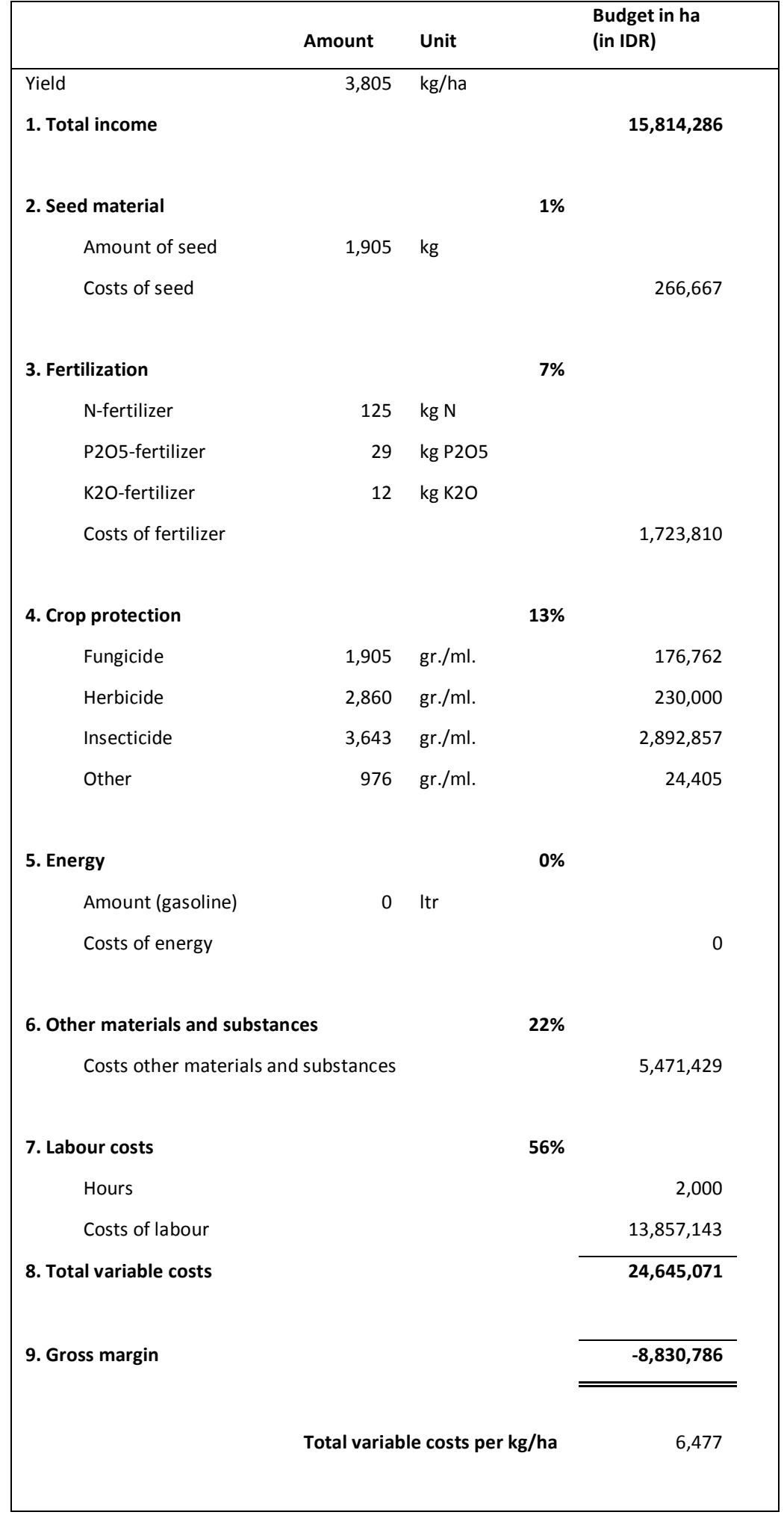




\subsection{Cereals}

Rice, wet season, average per hectare

$\mathrm{n}=$ 2

Average field size: 0.19 ha

\begin{tabular}{|c|c|c|c|c|c|c|}
\hline & Amount & Unit & $\begin{array}{l}\text { Budget in ha } \\
\text { (in IDR) }\end{array}$ & Median & Lowest & Highest \\
\hline Yield & 5,292 & $\mathrm{~kg} / \mathrm{ha}$ & & 5,292 & 4,583 & 6,000 \\
\hline 1. Total income & & & $21,483,333$ & $21,483,333$ & $20,166,667$ & $22,800,000$ \\
\hline 2. Seed material & & $2 \%$ & & & & \\
\hline Amount of seed & 51 & $\mathrm{~kg}$ & & 51 & 42 & 60 \\
\hline Costs of seed & & & 589,167 & 589,167 & 458,333 & 720,000 \\
\hline 3. Fertilization & & $43 \%$ & & & & \\
\hline $\mathrm{N}$-fertilizer & 60 & $\mathrm{~kg} \mathrm{~N}$ & & 60 & 52 & 67 \\
\hline P2O5-fertilizer & 11 & kg P2O5 & & 11 & 0 & 23 \\
\hline K2O-fertilizer & 11 & kg K2O & & 11 & 0 & 23 \\
\hline Costs of fertilizer & & & $13,674,028$ & $13,674,028$ & 230,000 & $27,118,056$ \\
\hline 4. Crop protection & & $3 \%$ & & & & \\
\hline Fungicide & 379 & gr./ml. & 358,575 & 358,575 & 0 & 717,150 \\
\hline Herbicide & 0 & gr./ml. & 0 & 0 & 0 & 0 \\
\hline Insecticide & 6,426 & gr./ml. & 515,711 & 515,711 & 52,222 & 979,200 \\
\hline Other & 306 & gr./ml. & 38,611 & 38,611 & 0 & 77,222 \\
\hline 5. Energy & & $0 \%$ & & & & \\
\hline Amount (gasoline) & 0 & Itr & & 0 & 0 & 0 \\
\hline Costs of energy & & & 0 & 0 & 0 & 0 \\
\hline 6. Other materials and sub & & $0 \%$ & & & & \\
\hline Costs other materia & substances & & 0 & 0 & 0 & 0 \\
\hline 7. Labour costs & & $52 \%$ & & & & \\
\hline Hours & & & 1,700 & 1,700 & 0 & 3,400 \\
\hline Costs of labour & & & $16,369,444$ & $16,369,444$ & $6,738,889$ & $26,000,000$ \\
\hline 8. Total variable costs & & & $31,547,236$ & & & \\
\hline \multirow[t]{2}{*}{ 9. Gross margin } & & & $-10,063,903$ & & & \\
\hline & \multicolumn{2}{|c|}{ Total variable costs per $\mathrm{kg} / \mathrm{ha}$} & 5,962 & & & \\
\hline
\end{tabular}




\section{References}

Annette Pronk, Lubbert van den Brink, Nikardi Gunadi, Uka Komara, Economics and Agronomics of Atlantic and Granola potato production in the dry season 2014 in West Java, vegIMPACT report 36, March 2017.

De Wit, C.T., 1992, On resource use efficiency in agriculture. Agricultural systems 40: 125-151.

Herman de Putter, Witono Adiyoga, Development of Permanent Vegetable Production Systems, vegIMPACT Internal report 4, March 2013.

Herman de Putter, Nikardi Gunadi, Uka, Romke Wustman, Huub Schepers, Economics and Agronomics of Atlantic and Granola potato cultivation in the dry season of 2013 in West Java, vegIMPACT internal report 10, March 2014.

Joanneke Spruijt, Marcel van der Voort, 2015, Kwantitatieve Informatie Akkerbouw en Vollegrondsgroenteteelt 2015, Praktijkonderzoek Plant \& Omgeving, Wageningen UR, ISSN 1571-3059.

Lubbert van den Brink, Nikardi Gunadi, Uka, Romke Wustman, Tonny K. Moekasa, Huib Hengsdijk, 2015, Agronomics and economics of potato production in West Java, Indonesia, Wet season 2013/2014, vegIMPACT External Report 17, Wageningen UR, The Netherlands.

Witono Adiyoga and Herman de Putter, 2017. Land tenure in the lowlands of Cirebon, Brebes and Pekalongan, Indonesia. vegIMPACT External Report 40. Wageningen UR, The Netherlands. 